\title{
Pure intelligent monitoring system for steam economizer trips
}

\author{
Firas Basim Ismail ${ }^{1 *}$, Khalid Hamzah Abed ${ }^{1}$, Deshvin Singh ${ }^{1}$, and Mohammad Shakir \\ $\mathrm{Nasif}^{2}$ \\ ${ }^{1}$ Power Generation Unit, Institute of Power Engineering (IPE), Universiti Tenaga Nasional, 43000 \\ Kajang, Selangor, Malaysia. \\ ${ }^{2}$ Mechanical Engineering Department, Universiti Teknologi Petronas, 32610 Tronoh, Malaysia.
}

\begin{abstract}
Steam economizer represents one of the main equipment in the power plant. Some steam economizer's behavior lead to failure and shutdown in the entire power plant. This will lead to increase in operating and maintenance cost. By detecting the cause in the early stages maintain normal and safe operational conditions of power plant. However, these methodologies are hard to be achieved due to certain boundaries such as system learning ability and the weakness of the system beyond its domain of expertise. The best solution for these problems, an intelligent modeling system specialized in steam economizer trips have been proposed and coded within MATLAB environment to be as a potential solution to insure a fault detection and diagnosis system (FDD). An integrated plant data preparation framework for 10 trips was studied as framework variables. The most influential operational variables have been trained and validated by adopting Artificial Neural Network (ANN). The Extreme Learning Machine (ELM) neural network methodology has been proposed as a major computational intelligent tool in the system. It is shown that ANN can be implemented for monitoring any process faults in thermal power plants. Better speed of learning algorithms by using the Extreme Learning Machine has been approved as well.
\end{abstract}

\section{Introduction}

Steam economizer is one of the main component in a power plant. Steams Economizers uses water as a heat transfer average tool and frequently also as the final point to reject heat into the atmosphere by evaporating inside cooling towers. Waterside problems develop in cooling water systems from scaling, corrosion, dirt and dust accumulation, and biological growth [1]. All these problems develop depending on the quality of water supply. The problems stated are commonly related with copper alloy type of steam economizer tubes. Rough solids in the cooling water could cause excess force inside the tubes. These forces create highly turbulent conditions at tube inlets, which results in destruction of protective passive films on the tube, causing a turbulent flow situation. Changes in flow direction and unfavourable design configurations of the waterbox are other factors that can contribute to high turbulence [2].

\footnotetext{
*Corresponding author: Firas@uniten.edu.my
} 
When there is a tube failure, abnormal changes can be observed in the related economizer variables such as economizer inlet pressure, economizer outlet temperature and feed water flow rate. As the tube failure is not detected and the unit continues operating, the readings of the variables deviates from its normal range. This deviation causes trips to occur when it reaches a certain maximum point. This causes frequent economizer trips which eventually leads to a power plant shutdown. Frequent shutdowns in a plant causes a significant increase in its operating and maintenance cost [3].

Conventional detection methods in power plants gives information on a particular problem only once serious damage has been done. Consequently prediction of economizer trips is important to maintain a normal and safe operational conditions. This is because when a trip is detected early, preventive measures can be taken by the plant operator to avoid further damage and shutdown. Eventually operating loss can be reduced by minimising repair cost and secondary damages [4]. Early detection is possible using intelligent monitoring system. Measurements from several sensors provide information on the operating system and its failures. This information can be used by an intelligent monitoring system for fault diagnosis [5]. One of the most commonly used intelligent systems for early fault detection is the Artificial Neural Network. The ANN approach is able to developed input-output simulation and forecasting models in situations that do not require modelling of the internal structure of the watershed [6]. The most commonly used family of feed-forward networks is a layered network in which neurons are organized into layers with connections strictly in one direction from one layer to another [7]. The most common type of feed-forward networks is the multilayer preceptor (MLP). An MLP, which has three types of layers: an input layer, an output layer and a hidden layer. Each neuron is in the hidden layer including its input signals after weighting them with the strengths of the relating connections from the input layer. The application of ANN in fault detection and diagnosis is based on model approximation and pattern recognition [8-10]. The pattern recognition method is a convenient approach to resolve the fault identification problem, for instance in determining the size of the fault [11]. Beale et al. provided a simple ANN model of steam cycle has been performed in 2002. The aim of that ANN model was just to show the capability of ANNs to predict the power output. That ANN model had just a few input parameters, and thereby the accuracy of the ANN was not so high [12]. In all proposed solutions, there has not been any intelligent monitoring system established particularly for detecting economizer trip in thermal power plants. Therefore it is crucial to develop a reliable intelligent monitoring system for prediction of thermal economizer trip. Mathematically simulated data were used by several researches instead of real plant data for training and validation, which is inaccurate for decision making. In most applications, the raw data are used directly for the training process. However this data is not always complete and the quality is limited. Therefore a data preparation framework is important for a thorough data analysis [13]. In this study, we concentrate on the steam economizer with 10 variables; only two of them were optimized and validated. The trips can be minimized using the fault detection and diagnostic (FDD) method and more specifically an artificial neural network (ANN) was the core diagnostic part in the system. This will have the effects on the power plant great performance and help the desperate industry to achieve a better outcomes and its production.

\section{Description of power plant}

This research was done based on real data collected from Manjung power plant in Malaysia. The unit consists of single reheat, controlled circulation type sub-critical pressure boiler. With a power generation of $700 \mathrm{MW}(\mathrm{e})$, the combustion system consists of a single furnace with balanced draught and tangential firing. 
In this research the power plant being considered consists of three sub-critical pressure boilers. Each boiler is a single reheat and controlled circulation type. The boiler is fired with pulverized coal to produce steam for the continuous generation of $700 \mathrm{MW}(\mathrm{e})$. The combustion system consists of a single furnace with balanced draught. Using direct tangential firing located in the furnace corners, the maximum heat input that can be attained is $40 \%$ of the Boiler Maximum Continuous Rate (BMCR). The capacity of steam generating for the unit is 172 bar with steam outlet temperature of $540^{\circ} \mathrm{C}$. The main auxiliary equipment includes two forced draft fans, two induced draft fans and two primary air fans which are centrifugal fans with control vanes at the inlet. Other components of the auxiliary equipment include two steam air preheaters, one piece of soot-blowing equipment, two electrostatic precipitators. The plant consists of low nitrogen oxide combustion burner system known as Over Fire Air (OFA) ports. The flue gas desulphurisation plant is controls the sulphur dioxide emission whereas the dust in the flue gas at the boiler outlet is removed by an Electro-Static Precipitator (ESP). Remote controlled fuel oil burners with high energy ignitions are used to start-up the boiler. Pulverized coal is able to undergo combustion at low firing rates using these burners.

\section{Data preparation framework}

Real-world data is often incomplete, inconsistent or lacking in certain behaviors or trends, and is likely to contain many errors. With proper data preparation framework, such issues can be resolved. The series of steps involved in this stage are:

\subsection{Data Capturing}

Data was captured from MNJ Power plant, Malaysia. More than 1000 variables were included in the data file. However, we have considered the most influent operational variables which can led to steam economizer trip. The time interval range when the fault has occurred is specified. Therefore, it is easy to recognize the variables through a sensitivity measure. The scope for this project is the boiler section whereby the unit is shut down due to the leakage of the boiler tubes. Based on the real data collected from the selected thermal power plant, the unit has been shut down from 25th April 2008 until 31 April 2008 and it is approximately about 5 days according to the outage.

\subsection{Data cleaning}

Large spreadsheet of data collected from sensors in power plants contains invalid values also known as noise. To avoid any error in the input data, the data has to undergo a few processes. The low pass filter method with moving average window was applied for this stage. The low pass filter with window size of 3 is applied on the data. The result of applying the low pass filter of type moving average window is acceptable in reducing the noise component that is affecting the raw data. However, small noise is still active. Probably, increasing the window size from 3 to larger sizes like 5 or 7 can have better performance in removing this noise component. But it is better to leave this option to be selected according the first impression obtained by the data visualization stage. Our choice is 3 because we aim at demonstrating the ANN decision validity even in case of further noise interfering with the data. 


\subsection{Data interpolation}

Piecewise polynomial interpolation was chosen. It has the capability of optimizing the smoothness of the fitted curve while passing through all the data points. Also, using it has the advantage of reducing the order of degree polynomial function because in each subinterval different polynomial is selected.

\subsection{Data normalization and segmentation}

Data were subject to data normalization in order to limit the values of the data between [-1 $+1]$. This step is needed because the neural network is trained on the relative change in the input not on the absolute values. Data was partitioned into two groups: 50\% training and 50\% checking. The only difference between the partitioned data and the raw data of the variable is the size of the data. This gives the opportunity to the ANN to capture all the information from the data and to reflect this information on its weights.

After undergoing the data processing procedure, the data was shortlisted into 10 important variables based on plant operator experience as listed in table 1 . The variables are shortlisted based on the critical sensors that contributed to the trip of that particular unit of boiler. Among all those 10 variables, there are several variables that had been identified contributed to the trip before the real shutdown. It is possible to reduce the number of variables to the most influential variables based on the data visualization of these 10 variables. Therefore variables number 3 and 5 were selected. These variables are very important because they caused immediate trip to the boiler.

Table 1. Selected variables for fault interpretation

\begin{tabular}{|c|l|l|}
\hline No & \multicolumn{1}{|c|}{ KKS } & \multicolumn{1}{|c|}{ Description } \\
\hline 1 & JMJG_U2_HAJ01CP501XV1 & RH LT R/HTR inlet pressure \\
\hline 2 & JMJG_U2_HAC01CT501X3V & Economizer inlet temperature \\
\hline 3 & JMJG_U2_HLA14CP501XV1 & RH Hot sec air pressure to burners [H12] \\
\hline 4 & JMJG_U2_HAC01CF501X5V & Steam flow \\
\hline 5 & JMJG_U2_HLA15CF501FL & Combustion air flow \\
\hline 6 & JMJG_U2_HAC61CF501X5V & Feed water flow \\
\hline 7 & JMJG_U2_HAD01CP501AXV1 & Boiler drum pressure \\
\hline 8 & JMJG_U2_HAH92CP501X3V & SH steam pressure \\
\hline 9 & JMJG_U2_HAH92CT501X3V & SH steam temperature \\
\hline 10 & JMJG_U2_HAC01CF501X5V & Total combined steam flow \\
\hline
\end{tabular}

\section{Design implementation and results of ims}

An efficient learning algorithm for single-hidden layer feed-forward neural network (SLFNs) called ELM is tested for training the data in steam economizer. Three different activation functions were applied to the weighted sum of its input. Training algorithms, learning rate, momentum coefficient, activation functions and number of neurons are the main neural network (NN) topology taken into consideration. A single hidden layer is used to be able to approximate arbitrarily with any system with continuous mapping from one finite space to another. The performance of each training results was indicated by their RMSE values. Based on this performance, the system is trained and validated. The variables selected are used as inputs for the IMS. The output is set to ' 0 ' or ' 1 ', representing normal economizer 
operation and faulty economizer operation respectively. In the first stage of designing the IMS, preliminary training is done to determine the optimal NN topology combination. Faulty data set is used as input to the IMS. Once optimal topology is obtained, the next stage involves basic training using $50 \%$ of the data. The RMSE results of the basic training process is compared with the calculated RMSE values of the optimal NN topology combination. This is to ensure a smaller or equal calculated RMSE values compared to the preliminary training process. The next stage involves the validation process using the remaining $50 \%$ of the data set. The optimal NN topology combination was applied to validate the real data set in order to determine the rapidness of the proposed IMS. Validation is also done for performance of the system during normal economizer operations. The capability of the IMS for specific trips was explored using the performance results obtained. It can observed from the table 2 the RMSE output for different activation function and different number of neurons in ANN hidden layer. Comparing the RMSE values in the table, the ANN topology with 20 neuron in its hidden layer performed better than the others. The activation functions performed differently for different number of neurons.

Table 2. RMSE values for different activation functions and number of neurons combination

\begin{tabular}{|c|c|c|c|}
\hline Activation Function & $\mathbf{5}$ & $\mathbf{1 0}$ & $\mathbf{2 0}$ \\
\hline sigmoid & 0.2344 & 0.2031 & 0.1194 \\
\hline sin & 0.3210 & 0.1393 & 0.1295 \\
\hline purelin & 0.2869 & 0.2301 & 0.1900 \\
\hline
\end{tabular}

The trained output of the proposed monitoring system is set to ' 0 ' and ' 1 ' for normal economizer operation and faulty economizer operation respectively. In between 0 to 1 , the lower threshold represents the point where economizer operation is normal whereas the upper threshold indicate the point of faulty operation.

The output of the system is displayed in figure 1. From the figure, we know that ELM performed better than conventional ANN algorithms. It has one-iteration learning, which gives this algorithm the capability of being executed for real time diagnostic. Furthermore ELM has a capability of universal approximation and is much simpler. The RMSE results obtained were very satisfying even with low number of neurons. The particular thermal economizer trip was successfully predicted by the proposed system.

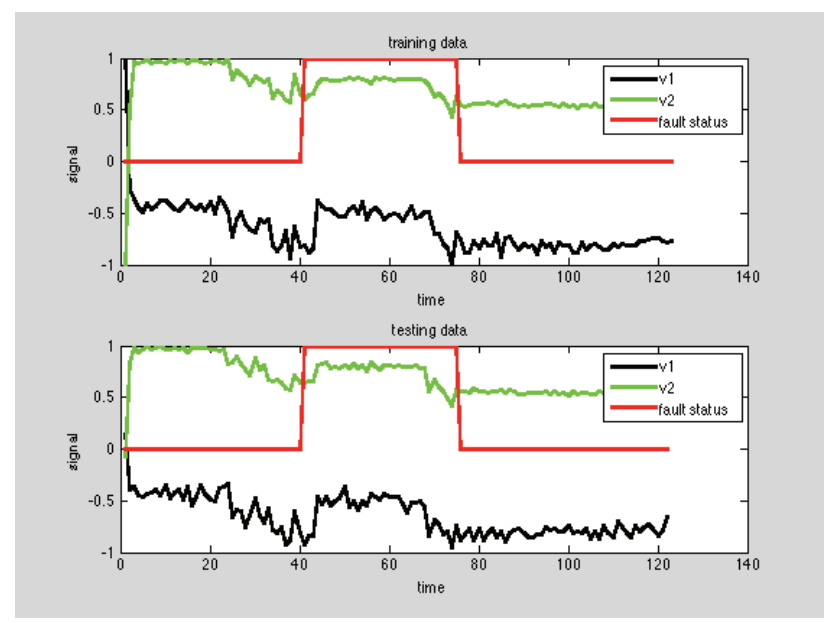

Fig. 1. ANN training data, and testing results for ELM. 


\section{Conclusion}

In this study, the early detection of economizer trips using intelligent monitoring system has been confirmed. Real data from power plant were used for training and validation purposes instead of mathematically simulated data. An integrated framework form economizer trip detection has been proposed in this study. The best ANN topology combination was achieved with RMSE of 0.1194 . The single layer feed-forward neural network was applied with ELM training algorithm in which it performed better than the conventional algorithms. The best activation function is the sigmoid function with 20 neurons in the hidden layer. ANN plays an important role in monitoring and analyzing the power plant's trips and faults. Using the monitoring system developed, trips can be detected earlier in order to maintain a continuous and safe plant operation. Further development of the proposed system can be considered in which online real time data is used for prediction of thermal power plant economizer tube leak trip.

The authors would like to express our gratitude to the power generation unit, institute of power engineering Universiti Tenaga Nasional (UNITEN) TNB Janamanjung and Ministry of Higher Education (MOHE), Malaysia for the opportunity to sponsorship this corresponding research.

\section{References}

1. Md. Mujibur and A. Kamal, Failure analysis of high temperature superheater tube of a pulverized coal-fired power station, Int. Scientific Conf. (2011)

2. Tallman P., Smith J., ASME Power (2004)

3. D. Fred, A. Dave, and E. Loren, Detection of tube leaks and their location using input/loss method Int. Joint Power Generation Conf. (2004)

4. D. Flynn, Thermal power plant simulation and control (The Institution of Electrical Engineers, 2003)

5. L. Fortuna, S. Graziani, A. Rizzo and M.G. Xibilia, Advances in Industrial Control (2007)

6. S.K. Chang and P.L. Hsu, Fault detection observer design for linear systems with unknown inputs, European Control Conference, 4, pp.1975-1980 (1993)

7. A.K. Jain, J. Mao and K.M. Mohiuddin, Computer, 29, pp. 31-44 (1996)

8. A. Lipnickas, Advanced information and knowledge processing, pp. 209-230 (2006)

9. Simani S., Fantuzzi C. and Patton R.J., Advances in Industrial Control, 1 (2003)

10. L. Zhou, R. E. Dickinson, Y. Tian, X. Zeng, Y. Dai, Z.-L. Yang, C. B. Schaaf, F. Gao, Y. Jin, A. Strahler, R. B. Myneni, H. Yu, W. Wu, M. Shaikh, Journal Of Geophysical Research, 108 (2003)

11. Simani S., Fantuzzi C. and Patton R.J., Advances in Industrial Control (2003)

12. Beale M.H., Hagan M.T., and Demuth, H.B., MathWorks (2014)

13. Messai, A. Mellit and I. Abdellani, Progress in Nuclear Energy, pp.8-21 (2015) 